\title{
Smart monitoring system for pressure regulator based on IOT
}

\author{
Aktham Hasan Ali ${ }^{1}$, Ahmed Hussein Duhis ${ }^{2}$, Nabeel Aad Lafta Alzurfi ${ }^{3}$, Mohannad Jabbar Mnati ${ }^{4}$ \\ ${ }^{1,4}$ Department of Electronic Technology, Institute of Technology Baghdad, Middle Technical University, Iraq \\ ${ }^{2}$ Electrical Power Engineering, Al-Furat Al-Awsat Technical University, Iraq \\ ${ }^{3}$ Department of Electrical Engineering, College of Engineering, University of Babylon, Iraq \\ ${ }^{4}$ Department of Electrical Energy, Metals, Mechanical Constructions and Systems, Ghent University, Belgium
}

\begin{tabular}{l}
\hline Article Info \\
\hline Article history: \\
Received Dec 27, 2018 \\
Revised Apr 4, 2019 \\
Accepted Apr 14, 2019 \\
\hline Keywords: \\
ESP8266 \\
IOT \\
NodeMCU \\
Power states \\
Pressure system \\
Thingspeak \\
Wi-fi
\end{tabular}

\begin{abstract}
In the last years, the interconnection of everyday instruments with internet using internet of thinks (IOT), is widely used as a monitoring and controlling in engineering as in smart cities, environmental, others. In this paper, a remote monitoring system of a pressure regulator is developed through an internet of thinks (IOT) communication tool, using MATLAB ${ }^{\circledR}$ programming platforms. The development of monitoring system for acquisition and communication corresponds to the NodeMCU as a microcontroller with ESP8266 for Wi-Fi connected. This system allows to monitor the reference of the pressure regulator and monitor the information of the transducers machine of the plant. Among the advantages of this type of development is that the server is free, and the development system is low cost, also with this type of projects can strengthen the infrastructure of laboratory equipment in engineering to be controlling in a way remote.
\end{abstract}

Copyright (C) 2019 Institute of Advanced Engineering and Science. All rights reserved.

Corresponding Author:

Aktham Hasan Ali,

Department of Electronic Technology,

Institute of Technology Baghdad,

Middle Technical University,

Baghdad, Iraq.

Email: akthamHasan@gmail.com

\section{INTRODUCTION}

The computers and the internet are practically dependent on human beings to collect information based on typing, pressing a button, taking a digital image or scanning a barcode. The problem is that people have limited time, attention and precision. Internet of Things $\left(\mathrm{I}_{\mathrm{O}} \mathrm{T}\right)$ is a concept that refers to the digital interconnection of everyday objects with the internet. Alternatively, the Internet of Things is the point in time in which more "things or objects" than people would connect to the Internet [1-8].

Currently there are different virtual platforms that allow an interaction between the user and the development systems through free or pre-paid services for different types of applications, one of them is Adafruit [9] that despite being a very useful tool , it is not so versatile when establishing a communication with devices that can connect to servers and public domains, because although it allows designing the components of the graphic control interface, it does not have a programming field in which users can send or receive values through its graphic components as it is a marketing license but not development.

With the Android Studio platform [10] you can develop communications between devices, because it has a wide range of possibilities to achieve this, such as integration with Google Cloud Platform, to access the different services that Google provides in the cloud, but because to use this service it is necessary to acquire an annual or monthly contract which leads to fixed costs, it is not used.

Thingspeak is an IOT platform that allows you to store and collect data using the protocol HTTP over the Internet or through a local area network [11]. It also allows the creation of applications sensor registration, positioning location applications, and a social network with notifications of the status updates. 
This platform is suitable for interacting with programs and packages Mathematics such as MatLab®, with hardware platforms such as Freescale ${ }$, Arduino ${ }^{\circledR}$ and other mobile devices besides its easy accessibility to exchange data between the plant and the device mobile, make this the most viable option. The university education institution observes the need for the equipment of the laboratories to be used remotely so that people from other places can access these through the internet and interact with different systems of control. It can be applied in the academic society because when sharing information in real time it gets a feedback of knowledge and a strong technological advance [12-21].

Within the engineering laboratories are conventional modules such as the level and temperature that could be handled remotely. Among others, there is a mechanism Pressure regulator which will be monitored and controlled through IOT technology. This regulator has a second order accumulator, with its respective control system (PLC), pressure sensor, I / P transducer, accumulator and pressure gauge.

The development of this paper is focused on the design new monitoring system for monitor the pressure and power states of a regulatory system through IOT that can be extended to other plants in the engineering laboratories of the universities. The full system is present in the Figure 1.

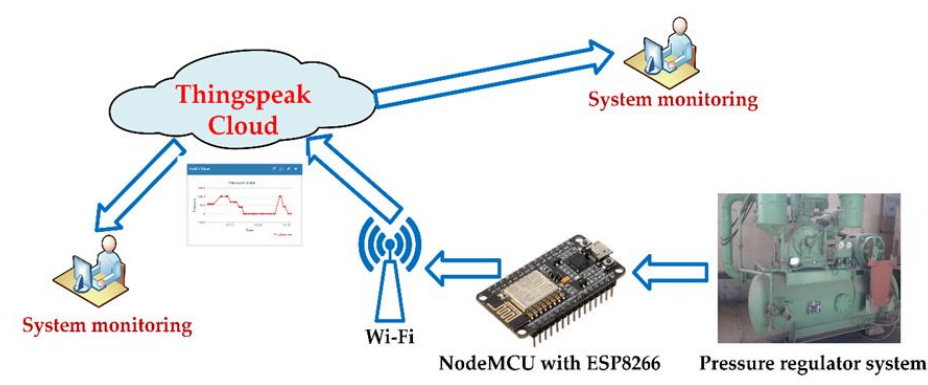

Figure 1. The Full smart system of pressure regulator based on IOT

The main parts of this work is, the NodeMCU with ESP8266 is an open source software that is very cheap, flexible, and has special-purpose data processing capabilities [22], The second part in the system is the pressure sensor G1/4 1.2Mpa [23], power states circuit based on the EL817 IC [24] and finally Thingspeak, Thingspeak is an open Internet code of application objects (IO) and the API to store and retrieve data from things using the HTTP protocol [11].

The aim of this work was to design new smart monitoring with low cost and safe for pressure regulator based on IOT. The rest of the paper is organized as follows: Section 2 presents the relevant related work, Section 3 presents main system material, Section 4 discusses the practical hardware and software system results, and finally, Section 5 presents conclusions.

\section{RELATED WORK}

In recent years, the academic research on smart monitoring and control techniques in all fields has increased. In this section, a similar smart monitoring research exhibition will be proposed in this research. The increase in smart homes, photovoltaic system in smart grid sensor networks and medical applications. In terms of monitoring software applications is how to monitor these devices by using smartphone or computer application software from long distance. Table 1 Present the related work for the last years according to the deferent fields application, type of monitoring, microcontroller, sensor and communication system.

Table 1. The related work for smart monitoring

\begin{tabular}{lllll}
\hline Related Work Reference & Application & $\begin{array}{l}\text { Type of } \\
\text { Com. system }\end{array}$ & $\begin{array}{l}\text { Type of } \\
\text { Controller }\end{array}$ & Type of Sensors \\
\hline A. H. Ali et al. [6], 2019 & Agricultural & LoRa & ESP32 & Voltage and Current \\
System
\end{tabular}




\section{SYSTEM MATERIALS}

\subsection{Pressure sensor G1/4 1.2Mpa}

The first part in the system is the pressure sensor G1/4 $1.2 \mathrm{Mpa}$ is shown in the Figure 2. This pressure measuring sensor we can use it in clean system and non-corrosive gas or liquid. The main proper tease is present in Table 2, the advantages of this sensor are small compact, easy to install for measuring, high quality with hall effect Sensor

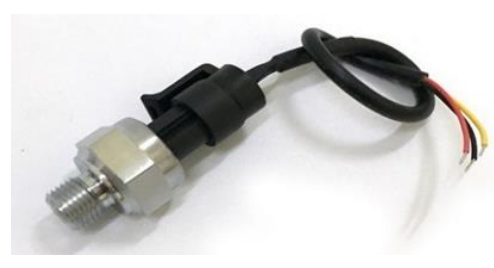

Figure 2. The pressure sensor G1/4 1.2Mpa [23]

Table 2. The main properties of the pressure sensor G1/4 1.2Mpa [23]

\begin{tabular}{cc}
\hline Parameter & Value \\
\hline Working voltage & $5 \mathrm{VDC}$ \\
Output voltage & $0.5-4.5 \mathrm{VDC}$ \\
Working current & $\leq 10 \mathrm{~mA}$ \\
Working TEMP. Range & $0-85 \mathrm{D}$ \\
Pressure range & $0-1.2 \mathrm{MPa}$ \\
Response Time & $\leq 2.0 \mathrm{~ms}$ \\
\hline
\end{tabular}

\subsection{Power states circuit}

The power states circuit, shown in Figure 3, this circuit is based on the EL817 IC [24]. The EL817 IC is a phototransistor photo coupler sensor used for isolated circuits. This device comes from the manufacturer according to the maximum furrowed current sensed $(5 \mathrm{~mA}), \mathrm{VCE}=5 \mathrm{~V}$ and Operating temperature up to $+110^{\circ} \mathrm{C}$.

The power states circuit indicates if the input current in the power circuit of the pressure regulator board can. The main voltage of the circuit, which is up to $250 \mathrm{~V}$ to $5 \mathrm{~V}$, is converted by high-value input resistance and there is an electrical circuit between the current of the main board and the NodeMCU board. Figure 3(a) shows the final power states circuit. Figure 3(b) exhibits the full circuit diagram of the electronic circuit in Figure 3(b).

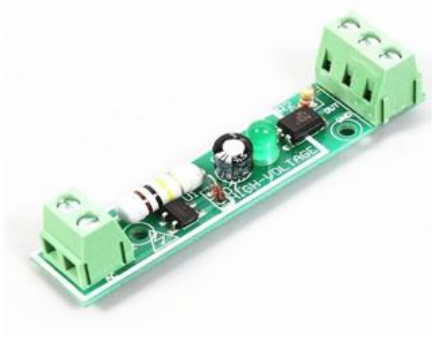

(a)

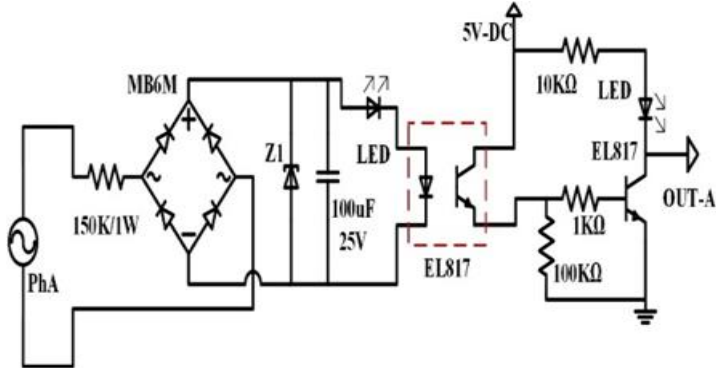

(b)

Figure 3. Power states circuit [24]: (a) hardware circuit and (b) the circuit diagram

\subsection{The microcontrol kit}

NodeMCU is one of the most relevant trends kits of the IoT [24]. NodeMCU in Figure 4 is the development kit based on the ESP8266 that makes life easier for those of us who want to develop connected devices to internet for monitoring and control. And within this technology it is worth mentioning a component that is becoming a rage among lovers of connected objects. This kit can be programed by using Arduino IDE, because, this program is an open-source software and support boards called NodeMCU. 


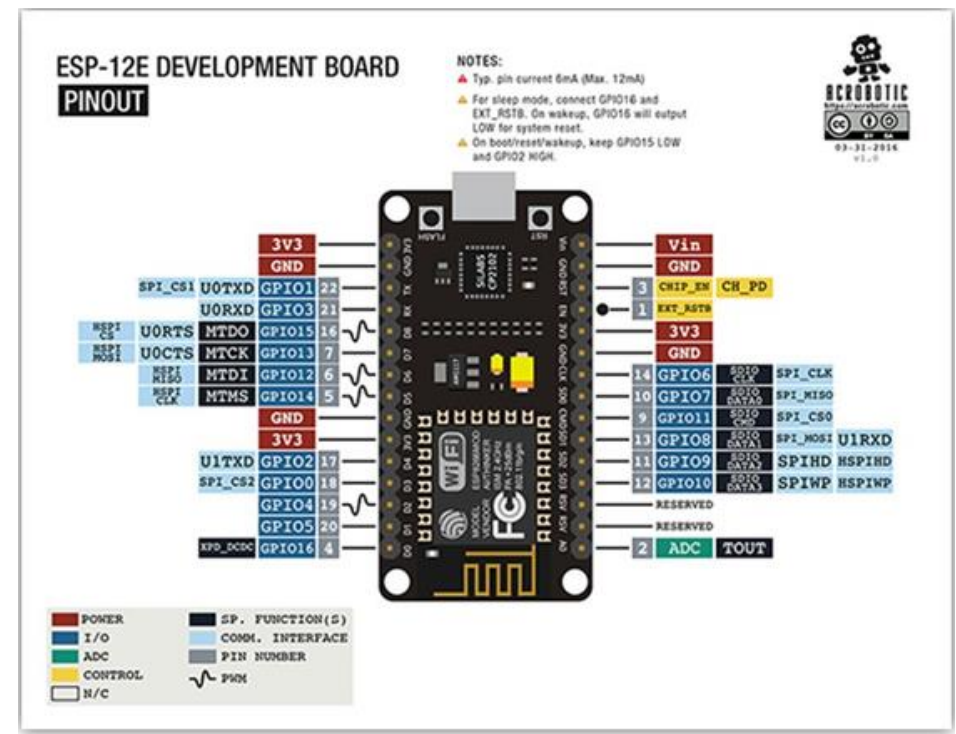

Figure 4. Bin diagram of NodeMCU with ESP8266 kit

The main properties of ESP8266, microcontroller cadence Tensilica Xtensa LX106 at 80 or 160 $\mathrm{MHz}$, external flash memory up to $4 \mathrm{MB}, 80 \mathrm{kB}$ of RAM, $10 \mathrm{GPIO}$ and (I2C, SPI, I2S, 1-Wire, UART) . The Wi-Fi network interface with WEP or WPA / WPA2 encryption and TCP / IPv4 protocols [27].

\subsection{The Thingspeak (IoT domain)}

The Internet of Things $\left(\mathrm{I}_{\mathrm{O}} \mathrm{T}\right)$ is a concept that refers to the digital interconnection of everyday objects with the internet. Alternatively, the Internet of Things is the point in time in which more "things or objects" than people would connect to the Internet.

Thingspeak is an open Internet code of application objects (IO) and the API to store and retrieve data from things using the HTTP protocol over the Internet or through a local area network." Thingspeak allows the creation of applications of sensor registration, tracking apps, and a social network of things with status updates. The applications of the Internet of Things cover a broad spectrum of our daily lives. One of the fields in which it is beginning to have and is expected to have great relevance is environmental sustainability. Figure 5 is shown the main screen of the Thingspeak platform, this platform is as open source platform available from MatLab.
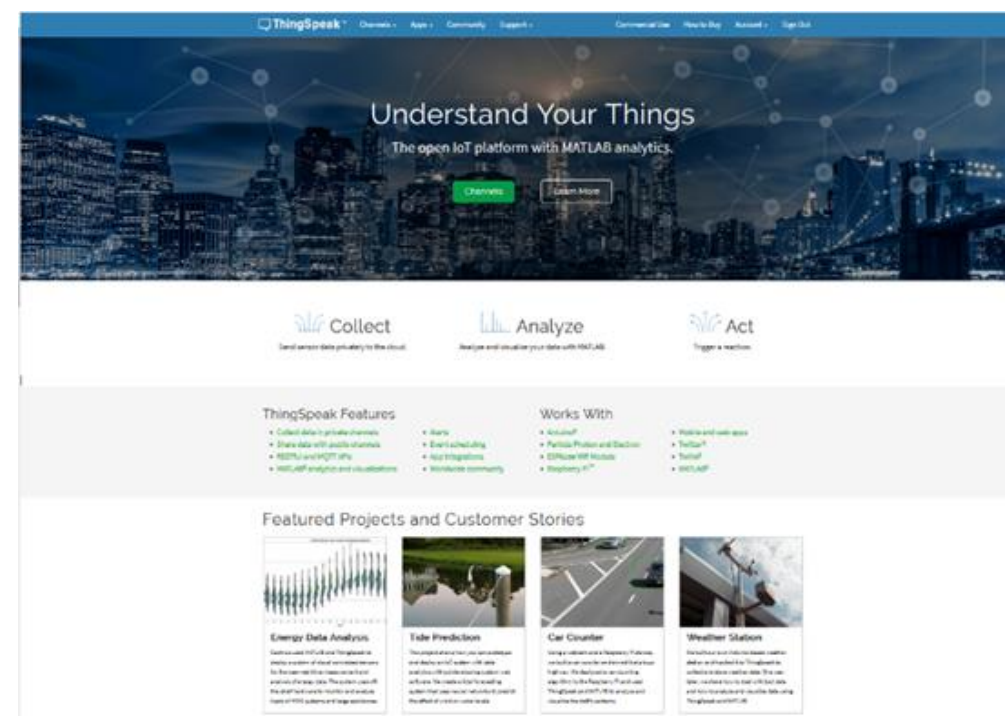

Figure 5. Main screen of Thingspeak platform [11] 


\section{RESULTS AND DISCUSSION}

The final designed circuit made in lab control kit for monitoring system for pressure regulator is presented in the Figure 6. It consists of the most important part NodeMCU with ESP8266 Wi-Fi technology, single-phase voltage control circuit to check the power states and pressure sensor to check the pressure states. The NodeMCU with ESP8266 development system was used, which communicates with the Thingspeak server through ESP8266. For the programming of the NodeMCU, an encoding was made from an interface of the same name. In this interface two libraries were declared that define the NodeMCU as a client and also enable communication with the ESP8266. Then the configuration parameters of the ESP8266 plate were declared, communication parameters were defined with the Thingspeak.

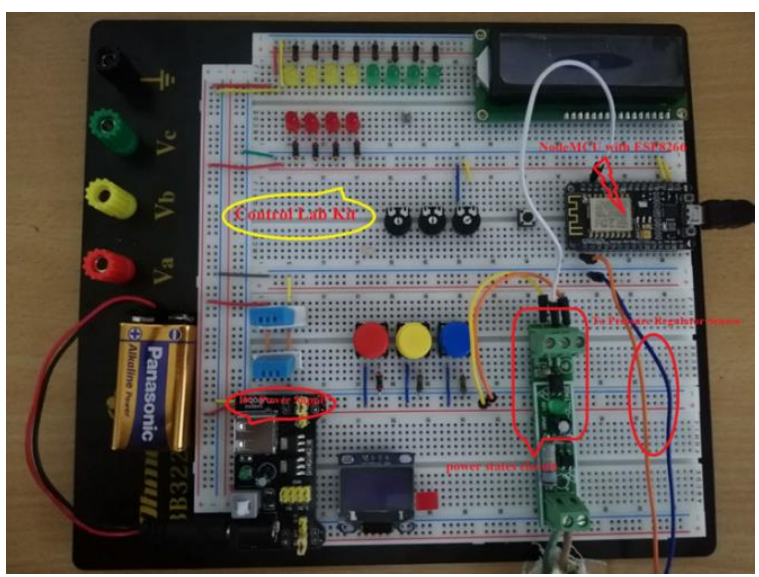

Figure 6. Final lab control practical system hardware of pressure regulator based on IOT

Figure 7 shows the flow chart of the of the monitoring program. The main programs used in this paper of NodeMCU kit with ESP8266 technologies, based on Arduino IDE open-source programs that are available on Arduino website.

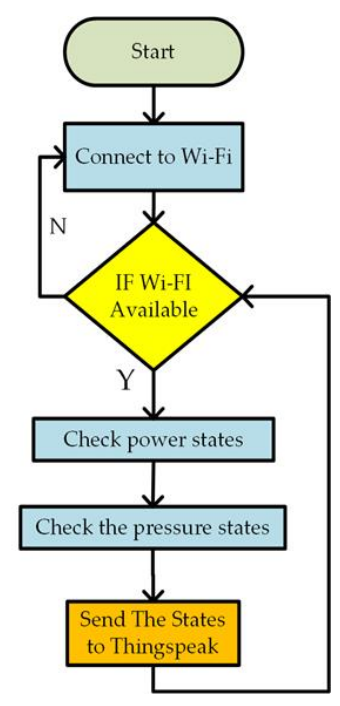

Figure 7. The flowchart of the full monitoring system

In the development of the system it was possible to obtain two visualization graphs in Figure 8 with a sampling time of 20 minute, in order to ensure a constant flow of information in the server, without traffic inconveniences. This sampling limits the sending of data at a higher speed, so it is not advisable to make applications where you need to monitor variables with rapid changes over time. 
Figure 8 present two charts, the first one (Field 1 chart) is to check the power states for the full system and the second one (Field 2 chart) is to check the pressure states. As we see in middle of the first chart for system power states, the power of the full system is (OFF) and the pressure output in second chart equal to zero also. The chart of the pressure states is present the output pressure when the power system is ON.

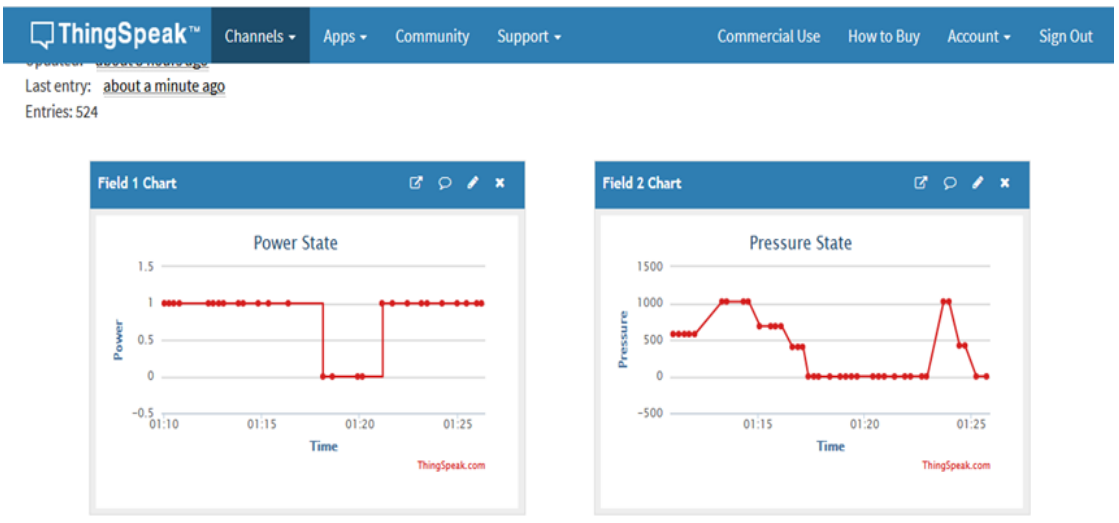

Figure 8 . The output result of pressure regulator system

\section{CONCLUSION}

In this paper, anew smart monitoring system designed for pressure regulator based on $\mathrm{I}_{\mathrm{O}} \mathrm{T}$ technology. The communication was developed between the ThingSpeak server and the NodeMCU interface with the pressure regulator system, monitoring the pressure transducers and controlling the system reference. A code was implemented with open source platform for the NodeMCU with ESP8266 development system with the ability to send data to the ThingSpeak server every 20seconds (maximum allowed rate).

The use of the NodeMCU with ESP8266 for full system is recommended for the connection process with the Thingspeak server since the IOT platform interacts specifically with this language, increasing the possibility of creating complex commands. The pressure module was monitored and controlled from a mobile device remotely by HTML programming, although there are payment servers that can be used for this development, a free service was used given that the scope is purely academic.

\section{REFERENCES}

[1] A. R. Biswas and R. Giaffreda, "IoT and cloud convergence: Opportunities and challenges," 2014 IEEE World Forum Internet Things, pp. 375-376, 2014.

[2] M. A. Ikram, et al., "Architecture of an IoT-based system for football supervision (IoT Football)," IEEE World Forum Internet Things, WF-IoT 2015 - Proc., pp. 69-74, 2015.

[3] A. Rghioui and A. Oumnad, "Internet of things: Surveys for measuring human activities from everywhere," International Journal of Electrical and Computer Engineering (IJECE), vol/issue: 7(5), pp. 2474-2482, 2017.

[4] P. Tan, et al., "Teaching Management System with Applications of RFID and IoT Technology," Educ. Sci., vol/issue: 8(1), pp. 26, 2018.

[5] M. J. Mnati, et al., "Smart monitoring and controlling of three phase photovoltaic inverter system using lora technology," 6th Eur. Conf. Ren. Energy Sys., Istanbul, Turkey, Jun 2018.

[6] A. H. Ali, et al., "A smart monitoring and controlling for agricultural pumps using LoRa IOT technology," Indonesian Journal of Electrical Engineering and Computer Science, vol/issue: 13(1), pp. 286-292, 2019.

[7] M. I. Younis and T. F. Hussein, "Design and Implementation of a Contactless Smart House Network System," International Journal of Electrical and Computer Engineering (IJECE), vol/issue: 8(6), pp. 4663-4672, 2018.

[8] L. Chhaya, et al., "IoT-Based Implementation of Field Area Network Using Smart Grid Communication Infrastructure," Smart Cities, vol/issue: 1(1), pp. 176-189, 2018.

[9] Adafruit. Available online: https://www.adafruit.com/.

[10] Android. Available online: https://developer.android.com/studio/.

[11] Thingspeak. Available online: https://thingspeak.com/.

[12] I. Doh and Y. H. Cho, "Passive flow-rate regulators using the parallel membrane valves rectifying dynamic inlet pressure," TRANSDUCERS 2009 - 15th Int. Conf. Solid-State Sensors, Actuators Microsystems, pp. 2278-2281, 2009. 
[13] L. Shkurti, et al., "Development of ambient environmental monitoring system through wireless sensor network (WSN) using NodeMCU and 'WSN monitoring," 2017 6th Mediterr. Conf. Embed. Comput. MECO 2017 - Incl. ECYPS 2017, Proc., pp. 1-5, Jun 2017.

[14] C. L. Chao, et al., "Intelligent gas pressure regulator control system," Proc. 29th Chinese Control Decis. Conf. CCDC 2017, pp. 2723-2728, 2017.

[15] D. Jiang, et al., "Design and experiment research of pressure control cavity on electronic oxygen regulator," 2017 8th Int. Conf. Mech. Aerosp. Eng. ICMAE 2017, pp. 656-660, 2017.

[16] M. J. Mnati, et al., "A smart voltage and current monitoring system for three phase inverters using an android smartphone application," Sensors (Switzerland), vol/issue: 17(4), 2017.

[17] A. Yun, et al., "Research on Gas Pressure Regulator Fault Diagnosis Based on Deep Confidence Network (DBN) Theory," IEEE, 2018.

[18] L. K. P. Saputra and Y. Lukito, "Implementation of air conditioning control system using REST protocol based on NodeMCU ESP8266," Proceeding 2017 Int. Conf. Smart Cities, Autom. Intell. Comput. Syst. ICON-SONICS 2017, pp. 126-130, 2018.

[19] J. Hasler and S. Shah, "Security Implications for Ultra-Low Power Configurable SoC FPAA Embedded Systems," J. Low Power Electron. Appl., vol/issue: 8(2), pp. 17, 2018.

[20] F. Yahya, et al., "A Top-Down Approach to Building Battery-Less Self-Powered Systems for the Internet-ofThings," J. Low Power Electron. Appl., vol/issue: 8(2), pp. 21, 2018.

[21] M. J. Mnati, et al., "A smart distance power electronic measurement using smartphone applications," 2017 19th European Conference on Power Electronics and Applications (EPE'17 ECCE EUROPE), pp. 1-11, 2017.

[22] NodeMCU. Available online: https://www.instructables.com/id/NodeMCU-ESP8266-Details-and-Pinout/.

[23] Seeed Technology, "Water Pressure Sensor G1/4 1.2Mpa - Datasheet," vol. 3, pp. 4-6, 2017.

[24] P. Configuration, "4 PIN DIP PHOTOTRANSISTOR PHOTOCOUPLER EL817-G Series," pp. 1-14, 2013.

[25] S. Vyas, "Access Control Application using Android Smartphone, Arduino and Bluetooth," vol/issue: 142(9), pp. 16-20, 2016.

[26] K. Kim and H. Myung, "Sensor node for remote monitoring of waterborne disease-causing bacteria," Sensors (Switzerland), vol/issue: 15(5), pp. 10569-10579, 2015.

[27] Espressif, “ESP8266EX Datasheet,” Espr. Syst. Datasheet, pp. 1-31, 2018. 